\title{
Análisis de fragilidad y riesgo de peritonitis en pacientes ancianos en diálisis peritoneal
}

\author{
Ana Isabel Aguilera-Flórez, Blanca Linares-Fano, Ana Cristina Alonso-Rojo, Juan Ramón Guerra-Ordoñez, \\ Aránzazu Sastre-López, $\mathbf{M}^{\mathrm{a}}$ del Carmen Barnes-Caso-Bercht, Mario Prieto-Velasco
}

Nefrología. Unidad de Diálisis. Complejo Asistencial Universitario de León. León. España

Como citar este artículo:

Aguilera-Florez AI, Linares-Fano B, Alonso-Rojo AC, Guerra-Ordóñez JR, Sastre-López A, Barnes-Caso-Bercht MC, Prieto-Velasco, M. Análisis de fragilidad y riesgo de peritonitis en pacientes ancianos en diálisis peritoneal. Enferm Nefrol. 2020 Abr-Jun;23(2):168-74

\section{Resumen}

Introducción: Los pacientes ancianos en diálisis peritoneal tienen mayor riesgo de presentar fragilidad, pérdida de autonomía, comorbilidad y disminución de calidad de vida.

Objetivo: evaluar la fragilidad, dependencia, depresión y calidad de vida, analizando la repercusión de la fragilidad sobre el tiempo de aprendizaje de la técnica y la aparición del primer episodio de peritonitis.

Material y Método: estudio descriptivo retrospectivo. Se incluyeron pacientes mayores de 70 años, desde septiembre 2016 a 2017, las peritonitis hasta final de 2018. Se estudiaron variables demográficas, modalidad dialítica, índices de Charlson, Barthel y escala de depresión de Yesavage Escala de fragilidad clínica, calidad de vida, tiempo de entrenamiento y primera peritonitis.

Resultados: Se incluyeron 25 pacientes, 56\% hombres, edad media $76,77 \pm 5,34$ años, el $72 \%$ estaban en diálisis peritoneal manual. La media del Charlson $7,88 \pm 2,06$, del Barthel $88,27 \pm 24,66$ y del Short form 12 health survey $32,96 \pm 8,61$. El $40 \%$ tenían algún grado de fragilidad, $24 \%$ depresión, el $28 \%$ precisaban ayuda o estaban institucionalizados. Tiempo medio

\section{Correspondencia:}

Ana Isabel Aguilera Flórez

Email: aaguilera@saludcastillayleon.es de entrenamiento en pacientes frágiles fue $16,77 \pm 7,93$ horas vs no frágiles $15,20 \pm 5,06(p=0,42)$. Se recogieron 16 episodios de peritonitis, repartidos al 50\%, tiempo medio de aparición frágiles $315,13 \pm 212,73$ días vs no frágiles $320,25 \pm 224,91 \quad(p=0,44)$.

Conclusiones: La mayoría de los pacientes realizan diálisis peritoneal manual de forma autónoma. Tienen un nivel de fragilidad bajo, no presentan depresión y gozan de buena calidad de vida para su edad. No existe diferencia en el tiempo de aprendizaje entre los dos grupos. Las peritonitis se reparten al $50 \%$ en frágiles y no frágiles.

PALABRAS CLAVE: diálisis peritoneal; ancianos; fragilidad; peritonitis; calidad de vida.

\section{Analysis of fragility and risk of peritonitis in elderly patients on peritoneal dialysis}

\section{Abstract}

Introduction: Elderly patients on peritoneal dialysis have a higher risk of presenting fragility, loss of autonomy, comorbidity and decreased quality of life. Objective: To assess frailty, dependency, depression and quality of life, analysing the repercussion of frailty on the learning time of the technique and the appearance of the first episode of peritonitis.

Material and Method: descriptive retrospective study. Patients older than 70 years were included, 
from September 2016 to 2017, episodes of peritonitis until the end of 2018. Demographic variables, dialysis modality, Charlson index, Barthel index, Yesavage geriatric depression scale, clinical fragility scale, quality of life scale, training time and first peritonitis were collected.

Results: 25 patients were included, $56 \%$ men, mean age $76.77 \pm 5.34$ years, $72 \%$ were on manual peritoneal dialysis. The mean value for the Charlson index was $7.88 \pm 2.06$, for Barthel index $88.27 \pm 24.66$ and for the Short form 12 health survey $32.96 \pm 8.61$. $40 \%$ had some degree of frailty, $24 \%$ depression, $28 \%$ needed help or were institutionalized. Average training time in fragile patients was $16.77 \pm 7.93$ hours versus nonfragile $15.20 \pm 5.06(p=0.42) .16$ episodes of peritonitis were collected, distributed to $50 \%$ between both groups. The mean time of appearance of fragile people was $315.13 \pm 212.73$ days versus $320.25 \pm 224.91$ days for non-fragile ones $(p=0.44)$.

Conclusions: Most of the patients perform manual peritoneal dialysis autonomously. They have low frailty levels, no depression and enjoy a good quality of life for their age. There is no difference in learning time between the two groups. Peritonitis is equally distributed in fragile and non-fragile patients.

KEYWORDS: peritoneal dialysis; elderly; fragility; peritonitis; quality of life.

\section{Introducción}

La enfermedad renal crónica (ERC) tiene una alta prevalencia en la población mundial, con un especial impacto en los ancianos ${ }^{1}$, ya que aumenta de forma progresiva con el envejecimiento (el $22 \%$ en mayores de 64 años, el $40 \%$ en mayores de 80 años) y con el incremento de factores de riesgo como son la enfermedad cardiovascular, la diabetes mellitus, la hipertensión arterial o la obesidad ${ }^{2,3}$.

Con los años, se producen cambios fisiológicos y aumenta el riesgo de enfermedades crónicas, discapacidad y dependencia, volviéndose las personas más frágiles ${ }^{4}$.

La fragilidad es un incremento de la vulnerabilidad a estresores de baja intensidad, producido por una alteración en múltiples e interrelacionados sistemas, que conduce a una disminución en la reserva homeostática y de la capacidad de adaptación del organismo, y lo predispone a eventos adversos de salud ${ }^{5}$. Es un fenotipo asociado con un mayor riesgo de caídas, discapacidad, hospitalización y muerte. Entre la población general, el $7 \%$ de los mayores de 65 años y el $40 \%$ de los mayores de 80 años cumplen los criterios de fragilidad 6 .

Los pacientes ancianos en diálisis tienen mayor riesgo de presentar algún grado de fragilidad, pérdida de autonomía, comorbilidad y disminución de su calidad de $v^{\text {vida }}{ }^{7}$. Este hecho, unido a otros factores relacionados con el proceso de envejecimiento como, discapacidad visual, auditiva, alteraciones cognitivas y perdida de destreza manual, pueden interferir en el aprendizaje de la técnica, aumentando el tiempo empleado en el entrenamiento, el riesgo de peritonitis y pudiendo afectar a su calidad de vida.

Evaluar de forma integral la capacidad de los pacientes para realizar la diálisis peritoneal (DP), es esencial para identificar las barreras que pueden dificultar el autocuidado y nos permitirá intervenir y prevenir complicaciones de forma precoz ${ }^{8}$.

Para ello, existen diferentes escalas de valoración validadas que facilitan la detección y seguimiento de problemas, así como la comunicación entre los diferentes profesionales que atienden al paciente anciano ${ }^{9}$.

El objetivo de este estudio fue evaluar la fragilidad, dependencia, depresión y calidad de vida de nuestros pacientes ancianos en diálisis peritoneal, y analizar la repercusión de la fragilidad sobre el tiempo de aprendizaje de la técnica y la aparición del primer episodio de peritonitis.

\section{Material y Método}

Se realizó un estudio observacional descriptivo de tipo retrospectivo. Los criterios de inclusión fueron: pacientes adultos mayores de 70 años con ERC estadío $V$ que estaban en programa de diálisis peritoneal en nuestro centro, entre el 1 de septiembre de 2016 y el 30 de septiembre de 2017, el seguimiento de los episodios de peritonitis se realizó hasta el 30 de diciembre de 2018, fecha en que se concluyó el estudio. Se excluyeron aquellos pacientes que eran totalmente dependientes en la realización de la técnica. Se consideró una edad igual o mayor a 70 años, porque el riesgo de discapacidad aumenta exponencialmente a partir de los 70 años $^{10}$.

Las variables estudiadas fueron: demográficas, soporte social, modalidad de diálisis, fragilidad, dependencia, 
comorbilidad, depresión, calidad de vida, tiempo de entrenamiento, tiempo a la primera peritonitis.

Se realizaron los siguientes cuestionarios y escalas:

- Escala de fragilidad clínica (Clinical Frailty Scale), resume el nivel general de aptitud o fragilidad de un adulto mayor después de haber sido evaluado por un clínico. Consiste en una escala de nueve puntos que clasifica a los pacientes en: robustos, bien sin enfermedad, bien con enfermedad controlada, vulnerable, fragilidad leve, moderada, grave, muy grave, enfermo terminal ${ }^{4}$.

Para evaluar el desempeño físico, se utilizó la Short Physical Performance Battery, (SPPB). Consiste en la realización de 3 pruebas:

- Test de equilibrio. Se realiza en tres posiciones: pies juntos, semitándem y tándem.

- Test de velocidad de la marcha. Se mide el tiempo empleado en caminar 2, 4 ó 6 metros a ritmo normal (se realizó sobre 4 metros).

- Test de levantarse de una silla. Se pide que cruce los brazos sobre el pecho e intente levantarse de la silla, tiene que realizarlo cinco veces ${ }^{5}$.

La puntuación total resulta de la suma de las tres pruebas, y oscila entre 0 (peor) y 12. Una puntuación por debajo de 10 indica fragilidad y un elevado riesgo de discapacidad, así como de caídas.

- El índice de Barthel (IB) es un instrumento que mide la capacidad de una persona para realizar diez actividades de la vida diaria (ABVD) consideradas como básicas, obteniéndose una estimación cuantitativa de su grado de independencia. El rango de posibles valores está entre 0 y 100, según la puntuación alcanzada se clasifica: 0-20 Dependencia total, 21-60 Dependencia severa, 61-90 Dependencia moderada, 91-99 Dependencia escasa, 100 independiente ${ }^{11}$.

- El índice de comorbilidad de Charlson (ICC). Es un sistema de evaluación de la esperanza de vida a los diez años, dependiendo de la edad en que se evalúa, y de las comorbilidades del sujeto ${ }^{12}$.

- La Escala de Depresión Geriátrica o test de Yesavage en su versión corta, es un cuestionario utilizado para el cribado de la depresión en personas mayores de 65 años. Consta de 15 preguntas en el que los participantes deben responder sí o no, con respecto a cómo se sintieron en la última semana. La puntación de 0-4 se considera normal; 5-8 indica depresión leve; 9-11 moderada; y $12-15$ severa $^{13}$.
- Escala de calidad de vida SF12 (Short Form 12 Health Survey), es un cuestionario de calidad de vida relacionado con la salud que evalúa la salud física y mental, consta de doce preguntas que miden ocho dominios de salud: Función Física, Función Social, Rol físico, Rol Emocional, Salud mental, Vitalidad, Dolor corporal, Salud General. Las opciones de respuesta son de tipo Likert y evalúan intensidad o frecuencia. El número de opciones de respuesta oscila entre tres y seis, dependiendo del ítem. La puntuación va de 0 y 100, a mayor puntuación mejor calidad de vida relacionada con la salud ${ }^{14}$.

La evaluación de las diferentes escalas, se realizó de manera directa a través de la aplicación de encuestas a cada paciente, empleando una media de tiempo de 15 minutos en cada cuestionario.

Los datos se obtuvieron de los registros de la historia clínica, hoja evolución clínica y Cronograma Enseñanza DPCA-DPA validado por la norma IS0-9001. Se creó una hoja de Excel para la recogida de datos y se trataron con el programa SPSS 15.

Las variables cualitativas se expresaron como valores absolutos y porcentajes y las cuantitativas en medias y desviación estándar. Se utilizó el coeficiente de correlación de Pearson para establecer la asociación lineal entre variables cuantitativas y la prueba $T$ de student para comparar medias, considerando como estadísticamente significativa una $p<0,05$.

Se solicitó el consentimiento informado a los pacientes, lo que garantizó la voluntariedad de participar en el estudio y la confidencialidad de la información que pudiera obtenerse. No se han incluido datos de carácter personal que pudieran identificar a los pacientes de forma directa o indirecta, respetando los principios éticos y universales, así como, las normas internacionales de protección de datos y la legislación española vigente.

\section{Resultados}

La muestra fue de 25 pacientes, el $56 \%$ fueron hombres, con una edad media de $76,77 \pm 5,34$ años, y un índice de comorbilidad de Charlson 7,88 $\pm 2,06$. El $72 \% \quad(n=18)$ realizaba DPCA y el $28 \%(n=11)$ en DPA. El $88 \%$ vivían en familia, el $8 \%$ vivían solos y un $4 \%$ institucionalizados. El $28 \%$ precisaban ayuda para realizar la DP. Los resultados de las puntuaciones medias en las diferentes escalas fueron: Î́ndice de Barthel $88,27 \pm 24,66$, fra- 
gilidad clínica 2,33 $\pm 1,89$, desempeño físico $10,6 \pm 3,66$,

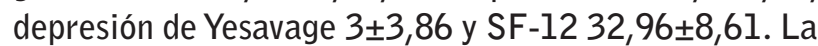
clasificación en las diferentes escalas se muestran en la Tabla 1.

Tabla 1. Clasificación de la población a estudio en función de las diferentes escalas empleadas.

\begin{tabular}{|l|l|l}
\hline Escala & Clasificación & $\begin{array}{l}\text { Porcentaje } \\
\text { (número) }\end{array}$ \\
\hline Índice de Barthel & $\begin{array}{l}\text { Independientes } \\
\text { Dependencia moderada } \\
\text { Dependencia severa }\end{array}$ & $\begin{array}{l}76 \%(19) \\
12 \%(3) \\
12 \%(3)\end{array}$ \\
\hline Grado de fragilidad & $\begin{array}{l}\text { No frágiles } \\
\text { Vulnerables }\end{array}$ & $60 \%(15)$ \\
\hline Fragilidad leve & $24 \%(6)$ \\
\hline Prueba de desempeño & Fragilidad grave & $8 \%(2)$ \\
\hline Test de Yesavo de discapacidad & y caídas & $48 \%(12)$ \\
\hline Test & $\begin{array}{l}\text { No depresión } \\
\text { Depresión leve }\end{array}$ \\
\hline & Depresión severa & $64 \%(16)$ \\
\hline
\end{tabular}

En el análisis del tiempo de entrenamiento y los episodios de peritonitis, en pacientes frágiles y no frágiles, no muestra diferencias estadísticamente significativas. Donde sí existe una diferencia significativa es en la edad, los pacientes frágiles son más añosos que los no frágiles. Los datos distribuidos por fragilidad se muestran en la Tabla 2.

Tabla 2. Comparativa del tiempo de entrenamiento y el tiempo a la primera peritonitis según la fragilidad.

\begin{tabular}{|c|c|c|c|}
\hline & No Frágiles & Frágiles & \multirow[t]{2}{*}{$\mathbf{p}$} \\
\hline & \multicolumn{2}{|c|}{ Media+ desviación estándar } & \\
\hline Edad & $74,89 \pm 4,84$ & $79,80 \pm 5,33$ & 0,016 \\
\hline $\begin{array}{l}\text { Tiempo de } \\
\text { entrenamiento (horas) }\end{array}$ & $15,20 \pm 5,06$ & $16,77 \pm 7,9$ & 0,425 \\
\hline $\begin{array}{l}\text { Tiempo hasta } \\
1^{\text {a }} \text { peritonitis (meses) }\end{array}$ & $320,25 \pm 224,91$ & $315,13 \pm 212,73$ & 0,44 \\
\hline
\end{tabular}

La asociación entre la fragilidad y la duración del entrenamiento, así como entre la fragilidad y el tiempo hasta la aparición del primer episodio de peritonitis, fue de $r=0,009$ y $r=-0,070$ respectivamente, no encontrando correlación lineal entre ellas.

En cuanto a la peritonitis, se recogieron 16 episodios, que se repartieron al $50 \%$. Los gérmenes causantes fue- ron el $75 \%$ Gram positivos, el $18,8 \%$ Gram negativos y el $6,2 \%$ presentó cultivo negativo. En la Figura 1, se muestran los gérmenes causantes de peritonitis en ambos grupos.

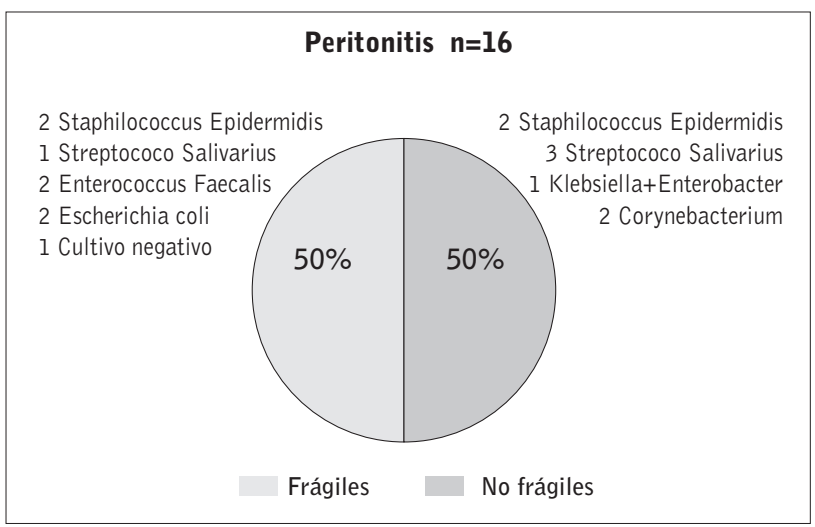

Figura 1. Gérmenes en el primer episodio de peritonitis.

\section{Discusión}

Las personas mayores forman el segmento de mayor crecimiento de la población de diálisis y la prevalencia de enfermedad renal crónica aumenta con la edad ${ }^{14}$. En la Comunidad de Castilla y León en 2016, año en el que comienza este estudio, el $53,9 \%$ de los pacientes incidentes tenían más de 70 años ${ }^{16}$.

Existe una gran variabilidad entre los individuos a medida que se envejece, mientras que algunas personas ancianas gozan de un buen funcionamiento físico y mental, otras tienen fragilidad o requieren apoyo considerable para satisfacer sus necesidades básicas ${ }^{17}$.

En este estudio observamos, que a pesar de la elevada comorbilidad que presentan, la mayoría de los pacientes ancianos en DP, son autónomos para las actividades de la vida diaria y capaces de realizar la diálisis de forma autónoma.

La fragilidad detectada fue baja, ya que la presentaban el $40 \%$ de los pacientes, a diferencia de otros estudios publicados, qué informan niveles de fragilidad más elevados del $54,6 \%$ y del $47,8 \% 18,19$.

En este estudio, la mayoría de los pacientes no presentaban depresión y gozaban de buena calidad de vida para su edad. Diferentes trabajos, informan también de buena calidad de vida del anciano en DP, similar o incluso mejor que en hemodiálisis ${ }^{20,21}$. 
Cabe destacar que, aquellos que alcanzaban puntuaciones más elevadas en la escala de calidad de vida, en general, son pacientes autónomos para las actividades de la vida diaria y no presentaban fragilidad ni depresión. Este dato, estaría en consonancia con otros estudios publicados, que muestran peores resultados en calidad de vida en pacientes frágiles en diálisis ${ }^{22,23}$.

La fragilidad es un síndrome frecuente en personas ancianas y su prevalencia aumenta de manera exponencial a medida que se envejece ${ }^{10}$, por eso, no es de extrañar que los pacientes frágiles de este estudio, tengan una edad significativamente más elevada, que los no frágiles.

En cuanto a las peritonitis no mostraron diferencia, presentando el mismo número de episodios entre los dos grupos, frágiles y no frágiles, con un tiempo de exposición a la primera peritonitis ligeramente inferior en los pacientes frágiles. Este resultado coincide con el hallazgo de otros estudios previos, que concluyen que la tasa de peritonitis no aumenta significativamente en los pacientes frágiles en DP ${ }^{24}$ y que la discapacidad física no es un factor de riesgo importante para la peritonitis en pacientes mayores en DP ${ }^{25}$.

Dado que, en la mayoría de las peritonitis, los gérmenes causantes fueron Gram positivos y siendo la contaminación la causa más probable, el reciclaje puede desempeñar un papel importante en la reducción de errores ${ }^{25,26}$ y en la prevención de episodios de peritonitis.

Respecto al tiempo de aprendizaje, fue ligeramente más elevado en los pacientes frágiles, aunque no hemos encontrado diferencia estadísticamente significativa entre ambos grupos.

Hay que tener en cuenta que las necesidades educativas de los pacientes ancianos pueden ser diferentes de los jóvenes 9 . El tiempo de entrenamiento debe ser individualizado en todos los pacientes y especialmente en los ancianos, por la posibilidad de presentar barreras para el aprendizaje como, trastornos cognitivos, disminución del estado de alerta, comprensión, deterioro funcional o déficit visual, entre otras, que pueden prolongar el tiempo de entrenamiento ${ }^{27}$.

Es posible, que el tiempo de entrenamiento de los pacientes frágiles, se haya podido ver reducido, debido a que algún paciente precisó que algún familiar le supervisara para la realización de la técnica. Este hecho y su carácter retrospectivo, pueden constituir una debilidad de este estudio.
En base a los resultados obtenidos podemos concluir que los pacientes ancianos de este estudio no presentaban una gran fragilidad, gozaban de una buena calidad de vida para su edad y eran autónomos para realizar la diálisis peritoneal; y si bien, los pacientes frágiles tienen mayor comorbilidad, depresión y peor movilidad, no necesitaron más tiempo para completar el entrenamiento, y aunque la primera peritonitis aparece antes, no llega a ser significativa la diferencia entre los dos grupos.

Recibido: 15-12-19

Revisado: 25-01-20

Modificado: 15-02-20

Aceptado: $30-02-20$

\section{Bibliografía}

1. Gorostidi M, Sánchez-Martínez M, Ruilope LM, Graciani A, de la Cruz JJ, Santamaría R et al. Prevalencia de la enfermedad renal en España: Impacto de la acumulación de factores de riesgo cardiovascular. Nefrología. 2018;38(6):606-15.

2. Ministerio de Sanidad Servicios Sociales e Igualdad. Documento marco sobre enfermedad renal crónica (ERC) dentro de la Estrategia de Abordaje a la Cronicidad en el SNS. Ministerio de Sanidad Servicios Sociales e Igualdad 2015 [Internet]. [Consultado 26 jul 2019]. Disponible en: https:// www.mscbs.gob.es/organizacion/sns/planCalidadSNS/pdf/Enfermedad_Renal_Cronica_2015.pdf.

3. Junta de Castilla y León. Consejería de Sanidad. Gerencia Regional de Salud. Dirección General de Asistencia Sanitaria. Proceso asistencial integrado para la prevencion y la atencion a las personas con Enfermedad Renal Cronica 2018 [Internet]. [Consultado 31 Jul 2019]. Disponible en: https://www.saludcastillayleon.es/profesionales/es/ procesos-asistenciales/procesos-asistenciales-gerencia-regional-salud/enfermedad-renal-cronica. ficheros/1367294-PAI Enfermedad Renal Crónica. pdf. 
4. Rockwood K, Song X, MacKnight C, et al. A global clinical measure of fitness and frailty in elderly people. CMAJ. 2005;173(5):489-95.

5. Ramos Cordero P (Coordinador). Guía de buena práctica clínica en geriatría. Fragilidad y nutrición en el anciano. Madrid: Sociedad Española de Geriatría y Gerontología;2014. p.7-15.

6. Berger JR, Hedayati SS. Renal Replacement Therapy in the Elderly Population. Clin J Am Soc Nephrol. 2012; 7(6):1039-46.

7. Portilla ME, Tornero F, Gil P. La fragilidad en el anciano con enfermedad renal crónica. Nefrología. 2016;36(6):609-15.

8. Hurst $H$, Figueiredo AE. The needs of older patients for peritoneal dialysis: Training and support at home. Perit Dial Int. 2015;35(6):625-9.

9. Fernández E, Estévez M. La valoración geriátrica integral en el anciano frágil hospitalizado: revisión sistemática. Gerokomos 2013;24(1):8-13.

10. Abizanda P, López-Torres J, Romero L, López M, Sánchez PM, Atienzar $P$, et al. Fragilidad y dependencia en Albacete (estudio FRADEA): razonamiento, diseño y metodología. Rev Esp Geriatr Gerontol. 2011;46(2):81-8.

11. Barrero C, García S, 0jeda A. Índice de Barthel (IB): Un instrumento esencial para la evaluación funcional y la rehabilitación. Plast \& Rest Neurol. 2005;4(1-2):81-5.

12. Índice de Comorbilidad de Charlson (ICC). Sociedad Andaluza de Medicina Intensiva y Unidades Coronarias (SAMIUC) 2018 [Internet]. [Consultado 29 Jul 2019]. Disponible en: http://www.samiuc.es/indice-de-comorbilidad-de-charlson-cci/.

13. Martínez de la Iglesia J, Onís Vilches MC, Dueñas Herrero R, Albert Colomer C, Aguado Taberné C, Luque Luque R. The Spanish version of the Yesavage abbreviated questionnaire (GDS) to screen depressive dysfunc-tions in patients older than 65 years. Medifam 2002;12(10):620-30.

14. Alonso J, Vilagut G, Ferrer M, Garin 0, Cunillera 0, Mompart $A$ et al. Versión española del Cuestionario de Salud SF-12. Institut Municipal d'Investigació
Mèdica (IMIM-IMAS) Unidad de Investigación en Servicios Sanitarios 2015 [Internet]. [Consultado 29 Mar 2019]. Disponible en: http://www.ser.es/ wp-content/uploads/2015/03/SF12_CUESTIONARIO.pdf.

15. Brown E, Johansson L. Dialysis Options for End-Stage Renal Disease in Older People. Nephron Clin Pract 2011;119(suppl 1):c10-c13.

16. Consejería de Sanidad de Castilla y León. Registro de Diálisis y Trasplante Renal de Castilla y León. Informe estadístico 2016 [Internet]. [Consultado 29 julio 2019]. Disponible en: https://www.saludcastillayleon.es/profesionales/es/donaciones-trasplantes/registro-dialisis-trasplante-renal-castilla-leon. ficheros/1225140-Informe\%20Estad\%C3\%ADstic0\%20a\%C3\%B10\%202016.pdf.

17. Organización Mundial de la Salud. Informe mundial sobre el envejecimiento y la salud. Organización Mundial de la Salud 2015 [Internet]. [Consultado 29 Mar 2019]. Disponible en: https://apps.who.int/ iris/bitstream/handle/10665/186471/WHO_FWC_ ALC_15.01_spa.pdf; jsessionid=98E109195F27FA4830F1DE3BB291DF7F? sequence $=1$.

18. Gil JM, Sánchez E, Rodríguez-Carmona A, Prieto M, Lanuza M, Paraíso $V$ et al. Registro de fragilidad en pacientes ancianos en diálisis peritoneal (DP). Estudio colaborativo. Comunicaciones orales presentadas en XLVII Congreso Nacional de la Sociedad Española de Nefrología (SEDEN). Madrid: SEDEN; 2018. p.107.

19. Ansur HN, Damasceno V0, Bastos MG. Prevalencia de fragilidad en pacientes con enfermedad renal crónica en tratamiento conservador y en diálisis. Braz. J. Nephrol 2012;34(2):153-60.

20. Brown $E$, Johansson $L$, Farrington $K$, Gallagher $H$, Sensky T, Gordon $F$ et al. Broadening Options for Long-term Dialysis in the Elderly (BOLDE): differences in quality of life on peritoneal dialysis compared to haemodialysis for older patients. Nephrol Dial Transplant. 2010;25(11):3755-63.

21. Lamping DL, Constantinovici N, Roderick $P$, Normand C, Henderson L, Harris $S$ et al. Clinical outcomes, quality of life, and costs in the North Thames Dialysis Study of elderly people on dialysis: a prospective cohort study. Lancet 2000; 356(9241):1543-50. 
22. Iyasere OU, Brown EA, Johansson $L$, Huson $L$, Smee $J$, Maxwell AP et al. Quality of life and physical function in older patients on dialysis: a comparison of assisted peritoneal dialysis with hemodialysis. Clin J Am Soc Nephrol 2016;11:423-30.

23. Nixon A, Bampouras T, Pendleton N, Mitra S, Brady $M$, Dhaygude A. Frailty is independently associated with worse health-related quality of life in chronic kidney disease: a secondary analysis of the Frailty Assessment in Chronic Kidney Disease study. Clin Kidney J. 2019;13(1):85-94.

24. Ng JK, Kwan BC, Chow KM, Cheng PM, Law MC, Pang WF, et al. Frailty in Chinese Peritoneal Dialysis Patients: Prevalence and Prognostic Significance. Kidney Blood Press Res. 2016;41(6):736-45.
25. Szeto CC. Peritoneal Dialysis-Related Infection in the Older Population. Perit Dial Int. 2015;35(6):659-62.

26. Dong J, Chen Y. Impact of the bag exchange procedure on risk of peritonitis. Perit Dial Int. 2010; 30(4):440-7.

27. Alarcón $R$, Roca $S$, Álvarez $M$, Álvarez G, Navarro $M J$, Pérez $F$, et al. Tratamiento sustitutivo renal en el anciano. Dial Traspl. 2015;36(2):87-92.

Este artículo se distribuye bajo una Licencia Creative Commons Atribución-NoComercial 4.0 Internacional. https://creativecommons.org/licenses/by-nc/4.0/

Open Access (C) () (9) 